Real Analysis Exchange

Vol. 23(2), 1997-1998, pp. 525-537

Julia Genyuk, Department of Mathematics, Ohio State University, Columbus, OH 43210, USA e-mail: genyuk@math.ohio-state.edu

\title{
A TYPICAL MEASURE TYPICALLY HAS NO LOCAL DIMENSION
}

\begin{abstract}
We consider local dimensions of probability measure on a complete separable metric space $X: \bar{\alpha}_{\mu}(x)=\varlimsup_{r \rightarrow 0} \frac{\log \mu\left(B_{r}(x)\right)}{\log r}, \underline{\alpha}_{\mu}(x)=\underline{\lim }_{r \rightarrow 0} \frac{\log \mu\left(B_{r}(x)\right)}{\log r}$. We show (Theorem 2.1) that for a typical probability measure $\underline{\alpha}_{\mu}(x)=0$ and $\bar{\alpha}_{\mu}(x)=\infty$ for all $x$ except a set of first category. Also $\underline{\alpha}_{\mu}(x)=0$ almost everywhere and with some additional conditions on $X$ there is a corresponding result for upper local dimension: in particular, we show that a typical measure on $[0,1]^{d}$ has $\bar{\alpha}_{\mu}(x)=d$ almost everywhere (Theorem 2.4).

There are similar results concerning "global" dimensions of probability measures. Theorems 2.2 and 2.3 show in particular that the Hausdorff dimension of a typical measure on any compact separable space equals 0 and the packing dimension of a typical measure on $[0,1]^{d}$ equals $d$.
\end{abstract}

\section{Introduction and Preliminaries}

Throughout this paper $X$ will denote a separable metric space. Let $\mathcal{P}(X)$ be the space of all Borel probability measures on $X$. Denote by $B_{r}(x)$ the open ball with center $x$ and radius $r$, and by $\bar{B}_{r}(x)$ the corresponding closed ball.

The Hausdorff dimension of a set $A$ is denoted by $\operatorname{dim} A$, and the packing dimension by $\operatorname{Dim} A$. For definitions see e.g. [5], [8]. We will use the "radius" definition of Dim, as in [8] and [4] as opposed to the "diameter" definition, since it allows to avoid some pitfalls in general metric spaces; for example, (4) is not true in general metric space with the "diameter" definition $([4])$.

Upper and lower local dimensions of a measure $\mu \in \mathcal{P}(X)$,

$$
\bar{\alpha}_{\mu}(x)=\varlimsup_{r \rightarrow 0} \frac{\log \mu\left(B_{r}(x)\right)}{\log r} \text { and } \quad \underline{\alpha}_{\mu}(x)=\varliminf_{r \rightarrow 0} \frac{\log \mu\left(B_{r}(x)\right)}{\log r},
$$

Key Words: measure, dimension, category

Mathematical Reviews subject classification: 28A33, 28A80

Received by the editors June 4, 1997 
have been extensively studied (see e.g. [3], [8], [12], [16] and many others). In particular, they are used to construct the so-called multifractal spectrum functions such as

$$
f_{\mu}(\alpha)=\operatorname{dim}\left\{x \in \operatorname{supp} \mu \mid \alpha_{\mu}(x)=\alpha\right\},
$$

where $\alpha_{\mu}(x)=\underline{\alpha}_{\mu}(x)=\bar{\alpha}_{\mu}(x)$ if they coincide. These limits are the same for closed and open balls.

The dimensions, $\underline{\alpha}_{\mu}(x)$ and $\bar{\alpha}_{\mu}(x)$, have been shown to coincide almost everywhere in some particular cases such as the ergodic invariant measures of smooth diffeomorphisms with nonzero Lyapunov exponents ([16]). If this happens, a measure is called regular. The multifractal spectrum was also computed for such constructions as graph-directed fractals and cookie-cutters (see e.g [8], where Olsen applies his general multifractal formalism to both cases). The question arises whether $\alpha_{\mu}(x)$ and $f(\alpha)$ can be used to describe more general situations. We will show that this is in fact not the case for a typical probability measure (in the sense of category).

Some relations between different notions of regularity are discussed in [14]. There are some results as well describing situations where $\underline{\alpha}_{\mu}(x) \neq \bar{\alpha}_{\mu}(x)$. For example, Taylor in [13] shows that this happens for super Brownian motion. Shereshevsky in [11] shows that under some conditions, if $\mu$ is an invariant measure of a smooth diffeomorphism, the set where $\underline{\alpha}_{\mu}(x) \neq \bar{\alpha}_{\mu}(x)$ is dense and has positive Hausdorff dimension. Finally, Haase in [7] shows that if $x \in X$, then for a typical measure $\mu \in \mathcal{P}(X)$ (that is, all measures up to a set of first category) $\underline{\alpha}_{\mu}(x)=0$, and if $x$ is a non-isolated point of $X$, then for a typical measure $\bar{\alpha}_{\mu}(x)=\infty$. Theorem 2.1 is basically a generalization of this result.

An interesting question, connected with this, is about the dimension of a typical measure, especially Hausdorff and packing dimension. The dimensions on other spaces have been explored before, such as the dimension of typical compact set or a graph of typical continuous function. For example, Hausdorff dimension of a typical compact subset of $\mathcal{R}^{d}$ is 0 and the upper entropy dimension is $d([6])$. We show later in this paper that the probability measures behave similarly in this matter.

We will use the following well-known relations.

$$
\begin{gathered}
\operatorname{dim}\left(\left\{x \in X \mid \underline{\alpha}_{\mu}(x) \leq \alpha\right\}\right) \leq \alpha . \\
\operatorname{Dim}\left(\left\{x \in X \mid \bar{\alpha}_{\mu}(x) \leq \alpha\right\}\right) \leq \alpha . \\
\text { If } \mu(A)>0, A \subseteq\left\{x \in X \mid \underline{\alpha}_{\mu}(x) \geq \alpha\right\}, \text { then } \operatorname{dim} A \geq \alpha . \\
\text { If } \mu(A)>0, A \subseteq\left\{x \in X \mid \bar{\alpha}_{\mu}(x) \geq \alpha\right\}, \text { then } \operatorname{Dim} A \geq \alpha .
\end{gathered}
$$


For the proof see e.g. [3], [8]. Note that (4) would not be true for all metric spaces if packing measure were defined using diameters, but true under some fairly general conditions.

A weak* topology on $\mathcal{P}(X)$ is characterized by the following proposition.

Proposition 1.1. Let $\mu,\left\{\mu_{n}\right\}_{n=1}^{\infty}$ be measures in $\mathcal{P}(X)$. Then the following statements are equivalent.

(a) $\mu_{n} \rightarrow \mu$ in weak* topology.

(b) $\lim _{n \rightarrow \infty} \int f d \mu_{n}=\int f d \mu$ for any bounded continuous function $f$.

(c) $\varlimsup_{n \rightarrow \infty} \mu_{n}(F) \leq \mu(F)$ for every closed set $F$.

(d) $\varliminf_{n \rightarrow \infty} \mu_{n}(G) \geq \mu(G)$ for every open set $G$.

0

(e) $\lim _{n \rightarrow \infty} \mu_{n}(A)=\mu(A)$ for every Borel set $A$ with boundary of $\mu$-measure

Proof. This is a version of slightly more general Theorem 6.1 in [9].

If $X$ is complete and separable, $\mathcal{P}(X)$ is also complete; so we can use an expression "a typical measure" to signify that all measures except a set of first category in $\mathcal{P}(X)$ have the desired properties.

Proposition 1.2. The probability measures with finite support are dense in $\mathcal{P}(X)$.

Proof. See Theorem 6.3 in [9].

It follows that $\mathcal{P}(X)$ is separable since $X$ is separable.

$\mathcal{P}(X)$ with weak* topology can be metrized in several ways. In particular for $X$ separable a Prokhorov metric $p$ can be used:

$$
\begin{aligned}
p(\mu, \nu)=\inf \{\epsilon>0 \mid \mu(A) & \leq \nu\left(A_{\epsilon}\right)+\epsilon \text { and } \\
\nu(A) & \left.\leq \mu\left(A_{\epsilon}\right)+\epsilon \text { for any Borel set } A\right\},
\end{aligned}
$$

where $A_{\epsilon}$ is the $\epsilon$-neighborhood of $A$ in $X$.

The inequalities (1)-(4) can also be used to establish a connection between the local and "global" dimensions of a measure. The latter can be computed in a number of ways. We will need the following definitions.

$$
\begin{aligned}
\operatorname{dim}^{*} \mu & =\inf \{\operatorname{dim} Y \mid Y \subseteq X, \mu(Y)=1\} \\
\operatorname{dim}_{*} \mu & =\inf \{\operatorname{dim} Y \mid Y \subseteq X, \mu(Y)>0\} . \\
\operatorname{Dim}^{*} \mu & =\inf \{\operatorname{Dim} Y \mid Y \subseteq X, \mu(Y)=1\} .
\end{aligned}
$$




$$
\begin{gathered}
\operatorname{Dim}_{*} \mu=\inf \{\operatorname{Dim} Y \mid Y \subseteq X, \mu(Y)>0\} . \\
\bar{C}(\mu)=\lim _{\delta \rightarrow 0} \inf \left\{\overline{\operatorname{dim}}_{B}(Y) \mid Y \subseteq X, \mu(Y) \geq 1-\delta\right\}, \\
\underline{C}(\mu)=\lim _{\delta \rightarrow 0} \inf \left\{\underline{\operatorname{dim}}_{B}(Y) \mid Y \subseteq X, \mu(Y) \geq 1-\delta\right\},
\end{gathered}
$$

where $\operatorname{dim} Y$ and $\operatorname{Dim} Y$ denote Hausdorff and packing dimensions correspondingly, and $\overline{\operatorname{dim}}_{B} Y, \underline{\operatorname{dim}}_{B} Y$ denote upper and lower box dimensions.

Ledrappier dimensions are defined as follows. Suppose $\mu$ is supported on a totally bounded set. Let $N_{\mu}(\epsilon, \delta)$ be the minimal number of balls of radius $\epsilon$ which cover a set of measure greater than $1-\delta$. Then

$$
\begin{aligned}
\bar{C}_{L}(\mu) & =\lim _{\delta \rightarrow 0} \varlimsup_{\epsilon \rightarrow 0} \frac{\log \left(N_{\mu}(\epsilon, \delta)\right)}{\log (1 / \epsilon)}, \\
\underline{C}_{L}(\mu) & =\lim _{\delta \rightarrow 0} \underline{\lim _{\epsilon \rightarrow 0}} \frac{\log \left(N_{\mu}(\epsilon, \delta)\right)}{\log (1 / \epsilon)} .
\end{aligned}
$$

(Here $\lim _{\delta \rightarrow 0}$ is the same as $\sup _{0<\delta<1}$ due to monotonicity.)

\section{Proposition 1.3.}

$$
\begin{gathered}
\operatorname{dim}^{*} \mu \leq \underline{C}_{L}(\mu) \leq \underline{C}(\mu) \text { and } \\
\bar{C}_{L}(\mu) \leq \bar{C}(\mu)=\operatorname{Dim}^{*} \mu .
\end{gathered}
$$

Proof. The last equality is proved in [12]; everything else is proved in [16].

Let us introduce also a few more global characteristics of a measure.

$$
\begin{aligned}
& \bar{C}_{L *}(\mu)=\lim _{\delta \rightarrow 1} \varlimsup_{\epsilon \rightarrow 0} \frac{\log \left(N_{\mu}(\epsilon, \delta)\right)}{\log (1 / \epsilon)}, \\
& \underline{C}_{L *}(\mu)=\lim _{\delta \rightarrow 1} \lim _{\epsilon \rightarrow 0} \frac{\log \left(N_{\mu}(\epsilon, \delta)\right)}{\log (1 / \epsilon)},
\end{aligned}
$$

(Here $\lim _{\delta \rightarrow 1}$ is the same as $\inf _{0<\delta<1}$.)

$$
\begin{gathered}
\bar{C}_{*}(\mu)=\inf \left\{\overline{\operatorname{dim}}_{B} Y \mid Y \subseteq X, \mu(Y)>0\right\} \text { and } \\
\underline{C}_{*}(\mu)=\inf \left\{\underline{\operatorname{dim}}_{B} Y \mid Y \subseteq X, \mu(Y)>0\right\} .
\end{gathered}
$$

\section{Proposition 1.4.}

$$
\underline{C}_{L *}(\mu) \leq \bar{C}_{L *}(\mu) \leq \bar{C}_{*}(\mu)=\operatorname{Dim}_{*} \mu .
$$


Proof. The first two inequalities are obvious, as is the inequality $\operatorname{Dim}_{*} \mu \leq$ $\bar{C}_{*}(\mu)$. For any $D>\operatorname{Dim}_{*} \mu$ there is a set $Y \subseteq X$ such that $\mu(Y)>0$ and $\operatorname{Dim} Y<D$. Note that $\operatorname{Dim} Y=\inf \left\{\sup _{i} \overline{\operatorname{dim}}_{B} A_{i} \mid Y \subseteq \cup A_{i}\right\}$ (for proof see e.g. [5], p.48; it is proved for $Y \in \mathbb{R}_{n}$ there, but the proof works for a general metric space with "radius" definition of packing dimension). Hence there is at most countable collection of sets $\left\{A_{i}\right\}$ such that $\sup _{i} \overline{\operatorname{dim}}_{B} A_{i}<D$ and $\mu\left(\cup A_{i}\right) \geq \mu(Y)>0$. It follows that there is an $A_{i}$ with $\mu\left(A_{i}\right)>0$, $\overline{\operatorname{dim}}_{B} A_{i}<D$. Hence $\operatorname{Dim}_{*} \mu \geq \bar{C}_{*}(\mu)$.

Proposition 1.5. Let $\mu$ be a probability measure on a compact space $X$. If for some $d$ there are numbers $c>0$ and $R>0$ such that $\mu\left(B_{r}(x)\right) \leq c r^{d}$ for all $x \in X, 0<r<R$, then $\underline{C}_{L *}(\mu) \geq d$.

Proof. Suppose that for some $\delta \varliminf_{\epsilon \rightarrow 0} \frac{\log N_{\mu}(\epsilon, \delta)}{\log (1 / \epsilon)}<d$. Then there are $a<d$ and $\epsilon_{l} \downarrow 0, \epsilon_{l}<R$ such that $N_{\mu}\left(\epsilon_{l}, \delta\right)<\epsilon_{l}^{-a}$ for all $l$. Hence for any $l$ there are $N_{\mu}\left(\epsilon_{l}, \delta\right)$ balls $\left\{B_{\epsilon_{l}}^{i}\right\}$ with $\mu\left(\cup_{i} B_{\epsilon_{l}}^{i}\right)>1-\delta$. But then

$$
1-\delta<\mu\left(\bigcup_{i} B_{\epsilon_{l}}^{i}\right) \leq c N_{\mu}\left(\epsilon_{l}, \delta\right) \epsilon_{l}^{d}<c \epsilon_{l}^{d-a} \text { for all } l,
$$

which contradicts the fact that $\epsilon_{l}^{d-a} \rightarrow 0$.

\section{Main Results}

Theorem 2.1. Let $X$ be a complete separable metric space. Then for a typical measure $\mu$ in $\mathcal{P}(X)$ there is a residual Borel set $A_{\mu}$ in $X$ such that for any $x \in A_{\mu}$ we have $\underline{\alpha}_{\mu}(x)=0$. If $X$ has no isolated points, then in addition we can have $\bar{\alpha}_{\mu}(x)=\infty$ for $x \in A_{\mu}$.

Proof. If $\mu_{n} \rightarrow \mu$ in weak* topology, then $\underline{\lim }_{n \rightarrow \infty} \mu_{n}(G) \geq \mu(G)$ for all open $G$ and $\overline{\lim }_{n \rightarrow \infty} \mu_{n}(F) \leq \mu(F)$ for all closed $F$ (Proposition 1.1). It follows that for fixed $x$ and $r$ the ratio $\frac{\log \mu\left(\bar{B}_{r}(x)\right)}{\log r}$ is lower semicontinuous and $\frac{\log \mu\left(B_{r}(x)\right)}{\log r}$ is upper semicontinuous with respect to $\mu$.

Consider closed balls first. Then

$$
\begin{aligned}
\Omega_{a, x, R} & =\left\{\mu \in \mathcal{P}(X) \mid \sup _{r<R} \frac{\log \mu\left(\bar{B}_{r}(x)\right)}{\log r}>a\right\} \\
& =\bigcup_{r<R}\left\{\mu \in \mathcal{P}(X) \mid \frac{\log \mu\left(\bar{B}_{r}(x)\right)}{\log r}>a\right\}
\end{aligned}
$$


is open for any $R>0, a>0, x \in X$. Now we want to show that $\Omega_{a, x, R}$ is dense in $\mathcal{P}(X)$. Let $\mu \in \mathcal{P}(X)$ and suppose that $\mu \notin \Omega_{a, x, R}$, that is, $\frac{\log \mu\left(\bar{B}_{r}(x)\right)}{\log r} \leq a$ for all $r<R$. Fix any $\epsilon>0$. If $\mu(\{x\}) \neq 0$, in case $X$ has no isolated points we can find a measure $\nu \in \mathcal{P}(X)$ such that $\rho(\mu, \nu)<\epsilon / 2$, and $\nu(\{x\})=0$. Otherwise let $\nu=\mu$. We construct $\mu_{\epsilon} \in \mathcal{P}(X)$ as follows. Pick some $s>a$ and some $r<R$ such that $\nu\left(B_{r}(x)\right)<\epsilon / 4$. Let $\mu_{\epsilon}\left(B_{r}(x)\right)=r^{s}$ and $\mu_{\epsilon}(A)=C \nu(A)$ for $A \subseteq X \backslash B_{r}(x)$, where $C=\left(1-r^{b}\right) /\left(1-\mu\left(B_{r}(x)\right)\right)$ so that $\mu_{\epsilon}(X)=1$. Then $\frac{\log \mu\left(\bar{B}_{r}(x)\right)}{\log r}>a$ and $\rho\left(\mu, \mu_{\epsilon}\right) \leq \epsilon / 2+\epsilon / 4+(C-1)\left(1-\mu\left(B_{r}(x)\right)\right)=$ $3 \epsilon / 4+\left(1-r^{b}\right)-1+\mu\left(B_{r}(x)\right)<\epsilon$. Hence $\Omega_{a, x, R}$ is dense in $\mathcal{P}(X)$.

Let $\left\{x_{i}\right\}_{i=1}^{\infty}$ be a countable dense subset of $X$. Then

$$
\Omega_{a, R}=\left\{\mu \in \mathcal{P}(X) \mid \sup _{r<R} \frac{\log \mu\left(\bar{B}_{r}\left(x_{i}\right)\right)}{\log r}>a \text { for all } i\right\}
$$

is a countable intersection of open dense sets, i.e. residual for any $a, R>0$. The same is true for $\Omega_{a}=\bigcap_{n} \Omega_{a, 1 / n}$. Now $\frac{\log \mu\left(\bar{B}_{r}(x)\right)}{\log r}$ is also lower semicontinuous with respect to $x$; so

$$
A_{a, \mu, R}=\left\{x \in X \mid \sup _{r<R} \frac{\log \mu\left(\bar{B}_{r}(x)\right)}{\log r}>a\right\}
$$

is open for any $a>0, R>0, \mu \in \mathcal{P}(X)$. For any fixed $\mu \in \Omega_{a}, A_{a, \mu, 1 / n}$ is open and dense (since $\left\{x_{i}\right\}_{i=1}^{\infty} \subseteq A_{a, \mu, 1 / n}$ ). Hence

$$
A_{a, \mu}=\bigcap_{n} A_{a, \mu, 1 / n}=\left\{x \in X \mid \sup _{r<1 / n} \frac{\log \mu\left(\bar{B}_{r}(x)\right)}{\log r}>a \text { for all } n \geq 1\right\}
$$

is residual in $X$. But

$$
A_{a, \mu} \subseteq\left\{x \in X \mid \varlimsup_{r \rightarrow 0} \frac{\log \mu\left(\bar{B}_{r}(x)\right)}{\log r} \geq a\right\}
$$

so

$$
\Omega_{a} \subseteq\left\{\mu \in \mathcal{P}(X) \mid \varlimsup_{r \rightarrow 0} \frac{\log \mu\left(\bar{B}_{r}(x)\right)}{\log r} \geq a \text { on a residual subset of } X\right\} .
$$

Now take the intersection $\Omega=\left(\bigcap_{n=1}^{\infty} \Omega_{1 / n}\right)$ and let the corresponding subset of $X$ for a fixed $\mu \in \Omega$ be $A_{\mu}=\bigcap_{n=1}^{\infty} A_{1 / n, \mu}$. This concludes the proof for the upper local dimension. 
The proof is similar for the lower local dimension. Using open balls, we can show that

$$
\bar{\Omega}_{b}=\left\{\mu \in \mathcal{P}(X) \mid \varliminf_{r \rightarrow 0} \frac{\log \mu\left(B_{r}(x)\right)}{\log r} \leq b \text { on a residual subset of } X\right\}
$$

is residual for any $b>0$. We need only to change sup to inf and reverse inequality signs in the proof above. (Also we will not need to consider the case $\mu(\{x\}) \neq 0$ separately, just take $\mu_{\epsilon}=r^{s}$ for some $s<b$ and some small enough $r<R$; so the condition that $X$ has no isolated points is not necessary here).

Take the intersection $\bar{\Omega}=\left(\bigcap_{n=1}^{\infty} \bar{\Omega}_{1 / n}\right)$ to conclude the proof for $\underline{\alpha}_{\mu}(x)$. Then $\Omega \cap \bar{\Omega}$ gives the residual set of measures for which $\underline{\alpha}_{\mu}(x)=0, \bar{\alpha}_{\mu}(x)=\infty$ for most $x \in X$.

The natural question arising next is whether we can take $A$ to be a set of positive measure. By (4), of course, we cannot have $\bar{\alpha}_{\mu}(x)>\operatorname{Dim} X$ on a set of positive measure; so we can hope only to get $\bar{\alpha}_{\mu}(x)=\operatorname{Dim} X$. We will show that we can in fact have $\underline{\alpha}_{\mu}(x)=0$ and $\bar{\alpha}_{\mu}(x)=\operatorname{Dim} X$ almost everywhere with some additional conditions on $X$ for the latter. To this end we need first to consider the "global" dimension of a typical measure.

Theorem 2.2. If $X$ is a compact separable metric space, a typical measure $\mu \in \mathcal{P}(X)$ has $\operatorname{dim}^{*} \mu=\underline{C}_{L}(\mu)=0$.

Theorem 2.3. Let $X$ be a compact separable metric space. Suppose there exists a probability measure $\lambda \in \mathcal{P}(X)$ which is positive on all open sets and has $\bar{C}_{L *}(\lambda) \geq d$. Then a typical measure $\mu \in \mathcal{P}(X)$ has $\operatorname{Dim}_{*} \mu \geq \bar{C}_{L *}(\mu) \geq d$.

Note. This is true, in particular, with $\lambda$ being a Lebesgue measure on $[0,1]^{d}$. We can also let $X$ be a self-similar fractal set of Hausdorff dimension $d$, with $\lambda$ being a Hausdorff measure $\mathcal{H}^{d}$. See Proposition 1.5.

We will need several lemmas to prove these theorems. In what follows, we will use the open balls in definition of $N_{\mu}(\epsilon, \delta)$, which does not change the limits.

Lemma 2.1. For $X$ a compact separable metric space, $N_{\mu}(\epsilon, \delta)$ is upper semicontinuous with respect to $\mu$.

Proof. Let $\mu_{n} \rightarrow \mu$ and $N_{0}(\epsilon, \delta)=\varlimsup_{n \rightarrow \infty} N_{\mu_{n}}(\epsilon, \delta)$. Since $N_{\mu_{n}}(\epsilon, \delta)$ is integer, taking subsequences if needed, we may assume that $N_{\mu_{n}}(\epsilon, \delta)=N_{0}(\epsilon, \delta)$. For 
any $N<N_{0}(\epsilon, \delta)$ the inequality $N_{\mu_{n}}(\epsilon, \delta)>N$ means that for any $N$ open balls $\left\{B_{\epsilon}\left(x_{i}\right)\right\}_{i=1}^{N}$ we have $\mu_{n}\left(\cup_{i=1}^{N} B_{\epsilon}\left(x_{i}\right)\right) \leq 1-\delta$. Hence

$$
\mu\left(\bigcup_{i=1}^{N} B_{\epsilon}\left(x_{i}\right)\right) \leq \varliminf_{n \rightarrow \infty} \mu_{n}\left(\bigcup_{i=1}^{N} B_{\epsilon}\left(x_{i}\right)\right) \leq 1-\delta \quad \text { from Proposition 1.1, }
$$

so $N_{\mu}(\epsilon, \delta)>N$. It follows that $N_{\mu}(\epsilon, \delta) \geq N_{0}(\epsilon, \delta)$.

Proof of Theorem 2.2. Since $N_{\mu}(\epsilon, \delta)$ is upper semicontinuous with respect to $\mu$ by Lemma 2.1 , so is $\frac{\log \left(N_{\mu}(\epsilon, \delta)\right)}{\log (1 / \epsilon)}$. Hence

$$
\Omega_{a, \epsilon_{0}, \delta}=\left\{\mu \in \mathcal{P}(X) \mid \inf _{\epsilon<\epsilon_{0}} \frac{\log \left(N_{\mu}(\epsilon, \delta)\right)}{\log (1 / \epsilon)}<a\right\}
$$

is open for any $a, \epsilon_{0}>0,0<\delta<1$. To show that $\Omega_{a, \epsilon_{0}, \delta}$ is dense in $\mathcal{P}(X)$, let $\mu \in \mathcal{P}(X)$. For any $\rho>0$ by Proposition 1.2 there is a measure $\mu_{\rho}$ with finite support such that $p\left(\mu, \mu_{\rho}\right)<\rho$. It means that for any $\delta, N_{\mu_{\rho}}(\epsilon, \delta)$ stays bounded as $\epsilon \rightarrow 0$; so for any $a>0, \epsilon_{0}>0$ there is an $\epsilon<\epsilon_{0}$ such that $\frac{\log N_{\mu_{\rho}}(\epsilon, \delta)}{\log (1 / \epsilon)}<a$. Now we have

$$
\Omega_{a, \delta}=\left\{\mu \in \mathcal{P}(X) \mid \varliminf_{\epsilon \rightarrow 0} \frac{\log \left(N_{\mu}(\epsilon, \delta)\right)}{\log (1 / \epsilon)} \leq a\right\} \supseteq \bigcap_{n=1}^{\infty} \Omega_{a, 1 / n, \delta}
$$

and this intersection is a dense $G_{\delta}$ set for any $a>0,0<\delta<1$. Taking intersections $\cap_{n=1}^{\infty} \cap_{m=1}^{\infty} \Omega_{1 / n, 1 / m}$, we get the result.

Note. The result concerning Hausdorff dimension can also be shown as follows. By Theorem 2.1 and (1) there is a residual Borel set $A \subseteq X$ with $\operatorname{dim} A=0$. It can be shown (see proof of Lemma 2 in [2]) that for any residual Borel set in $X$ there is a residual set of measures in $\mathcal{P}(X)$ concentrated on this set. But then for a typical measure $\mu \in \mathcal{P}(X)$ we have $\mu(A)=1$; so $\operatorname{dim}^{*} \mu=0$.

Lemma 2.2. For $X$ a compact separable metric space, $N_{\mu}(\epsilon, \delta)$ is left continuous with respect to $\epsilon$.

Proof. Let $\epsilon$ be a discontinuity point of $N_{\mu}(\epsilon, \delta)$ and let $\epsilon_{n} \uparrow \epsilon$. Let $N_{0}=$ $\lim _{\epsilon_{n} \rightarrow \epsilon} N_{\mu}\left(\epsilon_{n}, \delta\right.$ ) (which exists since $N_{\mu}(\epsilon, \delta)$ is a decreasing function of $\epsilon$ ). Since $N_{\mu}(\epsilon, \delta)$ is integer, for large $n$ we have $N_{\mu}\left(\epsilon_{n}, \delta\right)=N_{0}$. Pick any $N<N_{0}$ and any $N$ open balls $\left\{B_{\epsilon}\left(x_{i}\right)\right\}_{i=1}^{N}$. Then for large $n$ we have $\mu\left(\cup_{i=1}^{N} B_{\epsilon_{n}}\left(x_{i}\right)\right) \leq$ $1-\delta$. Hence $\mu\left(\cup_{i=1}^{N} B_{\epsilon}\left(x_{i}\right)\right)=\lim _{n \rightarrow \infty} \mu\left(\cup_{i=1}^{N} B_{\epsilon_{n}}\left(x_{i}\right)\right) \leq 1-\delta$; so $N_{\mu}(\epsilon, \delta)>$ $N$. By monotonicity $N_{\mu}(\epsilon, \delta)=N_{0}$. 
Lemma 2.3. If $X$ is a compact separable metric space, for any $\mu,\left\{\mu_{n}\right\}_{n=1}^{\infty}$ with $\mu_{n} \rightarrow \mu$, any $\epsilon>0,0<\delta<1,0<\nu<\min (\delta, 1-\delta)$ we have

$$
N_{\mu}(\epsilon+\nu, \delta+\nu) \leq \lim _{n \rightarrow \infty} N_{\mu_{n}}(\epsilon, \delta) .
$$

Proof. Let $N_{0}(\epsilon, \delta)=\varliminf_{n \rightarrow \infty} N_{\mu_{n}}(\epsilon, \delta)$. Since $N_{\mu_{n}}(\epsilon, \delta)$ is integer, taking subsequences we can assume that $N_{\mu_{n}}(\epsilon, \delta)=N_{0}(\epsilon, \delta)$. This means that for each $n$ there are $N_{0}(\epsilon, \delta)$ balls $B_{\epsilon}\left(x_{i}^{n}\right)$ such that $\mu_{n}\left(\cup_{i=1}^{N_{0}} B_{\epsilon}\left(x_{i}^{n}\right)\right)>1-\delta$.

Pick any $\nu$ as in the statement of the lemma. For large enough $n$ we have $p\left(\mu_{n}, \mu\right)<\nu$, where $p$ is a Prokhorov metric. For any Borel set $A$ we have then $\mu_{n}(A) \leq \mu\left(A_{\nu}\right)+\nu$. Let $A=\bigcup_{i=1}^{N_{0}} B_{\epsilon}\left(x_{i}^{n}\right)$. Then $A_{\nu}=\bigcup_{i=1}^{N_{0}} B_{\epsilon+\nu}\left(x_{i}^{n}\right)$; so we have

$$
\mu\left(\bigcup_{i=1}^{N_{0}} B_{\epsilon+\nu}\left(x_{i}^{n}\right)\right) \geq \mu_{n}\left(\bigcup_{i=1}^{N_{0}} B_{\epsilon}\left(x_{i}^{n}\right)\right)-\nu>1-\delta-\nu .
$$

Hence $N_{\mu}(\epsilon+\nu, \delta+\nu) \leq N_{0}(\epsilon, \delta)$.

Lemma 2.4. Let $X$ be a compact metric space. Suppose there exists a probability measure $\lambda \in \mathcal{P}(X)$ which is positive on all open sets and has $\bar{C}_{L *}(\lambda) \geq d$. Then the set of measures with $\bar{C}_{L *}(\mu) \geq d$ is dense in $\mathcal{P}(X)$.

Proof. Pick any $\mu \in \mathcal{P}(X)$ and $\eta>0$. Since $\lambda$ is positive on balls, using finite cover of $X$ by balls of radius $\eta / 2$ we can construct an $\eta$-partition $\left\{I_{k}^{\eta}\right\}_{k=1}^{K(\eta)}$ of $X$ with $\left|I_{k}^{\eta}\right|<\eta$ and $\lambda\left(I_{k}^{\eta}\right)>0$ for all $k$. Construct a new measure $\mu_{\eta}=\sum_{k} c_{k}^{\eta} \lambda_{k}^{\eta}$, where $\lambda_{k}^{\eta}$ is $\lambda$ restricted to $I_{k}^{\eta}$, and $c_{k}^{\eta}=\mu\left(I_{k}^{\eta}\right) / \lambda\left(I_{k}^{\eta}\right)$. Then $p\left(\mu, \mu_{\eta}\right) \leq \eta$. Suppose for some $\delta$

$$
\varlimsup_{\epsilon \rightarrow 0} \frac{\log N_{\mu_{\eta}}(\epsilon, \delta)}{\log (1 / \epsilon)}<d .
$$

Then there are $a<d$ and $\epsilon_{0}>0$ such that $N_{\mu_{\eta}}(\epsilon, \delta)<\epsilon^{-a}$ for all $\epsilon<\epsilon_{0}$. Hence for any such $\epsilon$ there are $N_{\mu_{\eta}}(\epsilon, \delta)$ balls $\left\{B_{\epsilon}^{i}\right\}$ with $\mu_{\eta}\left(\cup_{i} B_{\epsilon}^{i}\right)>1-\delta$. But then

$$
1-\delta<\mu_{\eta}\left(\bigcup_{i} B_{\epsilon}^{i}\right)=\sum_{k} c_{k}^{\eta} \lambda_{k}^{\eta}\left(\bigcup_{i} B_{\epsilon_{l}}^{i}\right) \leq C(\eta) \lambda\left(\bigcup_{i} B_{\epsilon}^{i}\right),
$$

where $C(\eta)=\max c_{k}^{\eta}$. It means that $N_{\lambda}\left(\epsilon, \delta_{0}\right) \leq \epsilon^{-a}$ for all $\epsilon<\epsilon_{0}$, where $\delta_{0}=1-(1-\delta) / C(\eta)$. Hence we have

$$
\varlimsup_{\epsilon \rightarrow 0} \frac{\log N_{\lambda}\left(\epsilon, \delta_{0}\right)}{\log (1 / \epsilon)} \leq a<d,
$$

which contradicts the assumption that $\bar{C}_{L *}(\lambda) \geq d$. 
Proof of Theorem 2.3. By Lemmas $2.1,2.3$ for any $\epsilon, \delta, \nu, \mu, \mu_{n} \rightarrow \mu$ as in the statements of the lemmas we have

$$
N_{\mu}(\epsilon+\nu, \delta+\nu) \leq \varliminf_{n \rightarrow \infty} N_{\mu_{n}}(\epsilon, \delta) \leq \varlimsup_{n \rightarrow \infty} N_{\mu_{n}}(\epsilon, \delta) \leq N_{\mu}(\epsilon, \delta)
$$

As $\epsilon$ or $\delta$ increase, $N_{\mu}(\epsilon, \delta)$ decreases. Fix $\mu \in \mathcal{P}(X)$. Consider all lines $\delta=\epsilon+\alpha$ on the plane with $\alpha$ rational. On each of these lines $N_{\mu}(\epsilon, \delta)$ is monotone; so it has countably many discontinuities there. All but countably many lines $\delta=\delta_{0}$ do not pass through any of these discontinuities. Denote the set of such $\delta_{0}$ 's by $D_{\mu}$. For each $\delta_{0} \in D_{\mu}$ there is a dense set $E_{\mu}(\delta)=\left\{\delta_{0}-\alpha \mid\right.$ $\alpha \in \mathbb{Q}\}$ such that for $\delta \in D_{\mu}, \epsilon \in E_{\mu}(\delta)$ we have $N_{\mu}(\epsilon+\nu, \delta+\nu) \rightarrow N_{\mu}(\epsilon, \delta)$ as $\nu \rightarrow 0$. It follows that for $\delta \in D_{\mu}, \epsilon \in E_{\mu}(\delta)$ all the inequalities above become equalities, which means $N_{\mu}(\epsilon, \delta)=\lim _{n \rightarrow \infty} N_{\mu_{n}}(\epsilon, \delta)$ for any $\mu_{n} \rightarrow \mu$, making $N_{\bullet}(\epsilon, \delta)$ continuous at $\mu$.

By Lemma 2.4 we can choose a countable dense set of measures

$$
\mathcal{M} \subseteq\left\{\mu \in \mathcal{P}(X) \mid \inf _{\delta} \varlimsup_{\epsilon \rightarrow 0} \frac{\log \left(N_{\mu}(\epsilon, \delta)\right)}{\log (1 / \epsilon)} \geq d\right\} .
$$

Let $D=\cap\left\{D_{\mu} \mid \mu \in \mathcal{M}\right\}$. $D$ contains all but countably many points of $(0,1)$. Pick any $\delta \in D$. Fix $\eta>0, \epsilon_{0}>0$. For any $\mu \in \mathcal{M}$ there is $\epsilon_{1}<\epsilon_{0}$ such that

$$
\frac{\log N_{\mu}\left(\epsilon_{1}, \delta\right)}{\log \left(1 / \epsilon_{1}\right)}>d-\frac{\eta}{2}
$$

By Lemma $2.2 N_{\mu}(\epsilon, \delta)$ is left continuous. Hence there is an interval $\left(\epsilon_{2}, \epsilon_{1}\right]$ on which $N_{\mu}(\epsilon, \delta)>d-\eta / 2$. But $E_{\mu}(\delta)$ is dense in $(0,1)$; so the set $E_{\mu}(\delta) \cap\left(0, \epsilon_{1}\right)$ is not empty. For any $\epsilon$ in this set, $\frac{\log \left(N_{\bullet}(\epsilon, \delta)\right)}{\log (1 / \epsilon)}$ is continuous at $\mu$; so there is an open neighborhood of $\mu$ such that for any measure $\nu$ in this neighborhood we have

$$
\left|\frac{\log N_{\nu}(\epsilon, \delta)}{\log (1 / \epsilon)}-\frac{\log \left(N_{\mu}(\epsilon, \delta)\right)}{\log (1 / \epsilon)}\right|<\frac{\eta}{2} .
$$

Hence by (5) and (6)

so

$$
\frac{\log N_{\nu}(\epsilon, \delta)}{\log (1 / \epsilon)}>d-\eta
$$

$$
\sup _{\epsilon<\epsilon_{0}} \frac{\log N_{\nu}(\epsilon, \delta)}{\log (1 / \epsilon)}>d-\eta \text {. }
$$

It follows that for any $\delta \in D, \eta, \epsilon_{0}>0$

$$
\Omega_{\epsilon_{0}, \delta, \eta}=\left\{\mu \in \mathcal{P}(X) \mid \sup _{\epsilon<\epsilon_{0}} \frac{\log \left(N_{\mu}(\epsilon, \delta)\right)}{\log (1 / \epsilon)}>d-\eta\right\}
$$


is an open dense set in $\mathcal{P}(X)$. Then

$$
\Omega_{\delta, \eta}=\left\{\mu \in \mathcal{P}(X) \mid \varlimsup_{\epsilon \rightarrow 0} \frac{\log \left(N_{\mu}(\epsilon, \delta)\right)}{\log (1 / \epsilon)} \geq d-\eta\right\} \supseteq \bigcap_{n=1}^{\infty} \Omega_{1 / n, \delta, \eta}
$$

is residual in $\mathcal{P}(X)$. Taking intersections $\cap_{n=1}^{\infty} \cap_{m=1}^{\infty} \Omega_{1 / n, 1 / m}$ concludes the proof.

Theorem 2.4. If $X$ is a compact separable metric space, then a typical measure $\mu \in \mathcal{P}(X)$ has $\underline{\alpha}_{\mu}(x)=0$ a.e. with respect to $\mu$. If there is a probability measure $\lambda \in \mathcal{P}(X)$ which is positive on all open sets and has $\bar{C}_{L *}(\lambda) \geq d$, then for a typical measure $\mu \in \mathcal{P}(X)$ also $\bar{\alpha}_{\mu}(x) \geq d$ a.e.

Proof. If $\operatorname{dim} \mu=0$, by (3) we have $\underline{\alpha}_{\mu}(x)=0$ a.e.

If $\operatorname{Dim}_{*} \mu \geq d$, by (2) we have $\bar{\alpha}_{\mu}(x) \geq d$ a.e. Theorems 2.3 and 2.2 now give the desired result.

\section{Example}

Let us now construct a simple example of a measure $\mu$ with $\underline{\alpha}_{\mu}(x)<\bar{\alpha}_{\mu}(x)$ a.e. The technique used here is common with this type of problem; see, for instance, Example 5.1 in [15] or a very detailed account of a similar example in [3].

Consider a Cantor-type set $F \subset(0,1)$ constructed as follows. Let $\left\{a_{i}\right\}_{i=1}^{\infty}$ be a sequence of positive integers. Let $p_{1}, p_{2}$ be positive integers and $r_{1}, r_{2}>0$ such that $p_{1} r_{1}<1, p_{2} r_{2}<1$. Replace $I_{0}=[0,1]$ by $p_{1}$ disjoint intervals of length $r_{1}$. Repeat this with each of the resulting intervals and so on. Do this $a_{1}$ times; then do the same with parameters $p_{2}, r_{2} a_{2}$ times; then again with $p_{1}, r_{1}$ $a_{3}$ times and so on. Let $\mu$ be a probability measure which is equally divided between all intervals on each step. Let $c_{k}=\sum_{i=1}^{k} a_{i}, b_{k}=\sum_{i=1}^{k} a_{2 i}, d_{k}=$ $\sum_{i=1}^{k} a_{2 i-1}$. We will denote by $I_{n}$ any interval of construction after $c_{n}$ steps.

Then for the length of intervals we have

$$
\left|I_{2 k}\right|=r_{1}^{d_{k}} r_{2}^{b_{k}}, \quad\left|I_{2 k+1}\right|=r_{1}^{d_{k+1}} r_{2}^{b_{k}},
$$

and for the measure

$$
\mu\left(I_{2 k}\right)=p_{1}^{-d_{k}} p_{2}^{-b_{k}}, \quad \mu\left(I_{2 k+1}\right)=p_{1}^{-d_{k+1}} p_{2}^{-b_{k}} .
$$


Hence

$$
\begin{aligned}
& \alpha_{2 k}:=\frac{\log \mu\left(I_{2 k}\right)}{\log \left|I_{2 k}\right|}=\frac{-d_{k} \log p_{1}-b_{k} \log p_{2}}{d_{k} \log r_{1}+b_{k} \log r_{2}} \\
& =-\frac{\log p_{2}}{\log r_{2}} \frac{\frac{d_{k}}{b_{k}} \frac{\log p_{1}}{d_{k}}+1}{\frac{\log p_{2}}{b_{k}}+1}, \\
& \alpha_{2 k+1}:=\frac{\log \mu\left(I_{2 k+1}\right)}{\log \left|I_{2 k+1}\right|}=-\frac{\log p_{2}}{\log r_{2}} \frac{\frac{d_{k+1}}{b_{k}} \frac{\log p_{1}}{\log p_{2}}+1}{\frac{d_{k+1}}{b_{k}} \frac{\log r_{1}}{\log r_{2}}+1} .
\end{aligned}
$$

Suppose we choose $\left\{a_{i}\right\}$ so that $\varlimsup_{k \rightarrow \infty}\left(d_{k+1} / b_{k}\right)>\varliminf_{k \rightarrow \infty}\left(d_{k} / b_{k}\right)$ (that is, $\left.\varlimsup_{k \rightarrow \infty}\left(a_{2 k+1} / \sum_{i=1}^{k} a_{2 i}\right)>0\right)$. Then we have

$$
\underline{\alpha}=\varliminf_{k \rightarrow \infty} \frac{\log \mu\left(I_{2 k}\right)}{\log \left|I_{2 k}\right|}<\varlimsup_{k \rightarrow \infty} \frac{\log \mu\left(I_{2 k+1}\right)}{\log \left|I_{2 k+1}\right|}=\bar{\alpha}
$$

For $x \in F$ let now $\left\{I_{n}\right\}_{n=0}^{\infty}$ be a sequence of intervals converging to $x$. Let $\epsilon_{k}=\left|I_{2 k}\right|$. We have $B_{\epsilon_{k}}(x) \supseteq I_{2 k} ;$ so

$$
\frac{\log \mu\left(B_{\epsilon_{k}}(x)\right)}{\log \epsilon_{k}} \leq \frac{\log \mu\left(I_{2 k}\right)}{\log \left|I_{2 k}\right|} .
$$

Hence $\varliminf_{\epsilon \rightarrow 0} \frac{\log \mu\left(B_{\epsilon}(x)\right)}{\log \epsilon} \leq \underline{\alpha}$. After $c_{2 k+1}$ steps the distance between two intervals of construction is at least $c\left|I_{2 k+1}\right|$, where $c$ is the minimal distance between intervals after the first step. Let $\delta_{k}=c\left|I_{2 k+1}\right|$. Then $F \cap B_{\delta_{k}}(x) \subseteq I_{2 k+1}$; so

$$
\frac{\log \mu\left(B_{\delta_{k}}(x)\right)}{\log \delta_{k}} \geq \frac{\log \mu\left(I_{2 k+1}\right)}{\log c\left|I_{2 k+1}\right|} .
$$

Hence $\varlimsup_{\epsilon \rightarrow 0} \frac{\log \mu\left(B_{\epsilon}(x)\right)}{\log \epsilon} \geq \bar{\alpha}$.

The author thanks Professor G. Edgar for introducing her to this topic and for his patient guidance of her research.

\section{References}

[1] P. Billingsley, Convergence of Probability Measures (Wiley, 1968).

[2] J. B. Brown, Baire category in spaces of probability measures, Fund. Math, 96 (1977), 189-193. 
[3] C. D. Cutler, Measure disintegration with respect to $\sigma$-stable monotone indices and the pointwise representation of packing dimension, Rend. Circ. Mat. Palerno(2) Suppl., 28 (1992), 319-339.

[4] C. D. Cutler, The density theorem and Hausdorff inequality for packing measure in general metric spaces, Illinois J. Math, 39 (1995), 676-694.

[5] K. Falconer, Fractal Geometry: Mathematical Foundations and Applications (Wiley, 1990).

[6] P. M. Gruber, Dimension and structure of typical compact sets, continua and curves, Monatsh. Math, 108 (1989), 149-164.

[7] H. Haase, A survey on the dimension of measures, In Topology, Measures and Fractals, Math. Res. 66 (Akademie Verlag, 1992).

[8] L. Olsen, A multifractal formalism, Adv. in Math. 116 (1995), 82-196.

[9] K .R .Parthasarathy, Probability Measures on Metric Spaces (Academic Press, 1967).

[10] Ya. B. Pesin. On rigorous mathematical definitions of correlation dimension and generalized spectrum for dimensions, J. Stat. Phys., 71 (1993), 529-547.

[11] M. A. Shereshevsky, A complement to Young's theorem on measure dimension: the difference between lower and upper pointwise dimensions, Nonlinearity 4 (1991), 15-25.

[12] M. Tamashiro, Dimensions in a separable metric space, Kyushu J. Math 49 (1995), 143-162.

[13] S. J. Taylor, Super Brownian motion is a fractal measure for which the multifractal formalism is invalid, Fractals 3 (1995), 737-746.

[14] S. J. Taylor, The fractal analysis of Borel measures in $\mathbb{R}^{d}$, J. Fourier Anal. Appl., Special Issue (1995), 553-568.

[15] S. J. Taylor and C. Tricot, The packing measure of rectifiable subsets of the plane, Math. Proc. Camb. Phil. Soc., 99 (1986), 285-296.

[16] L-S. Young, Dimension, enthropy and Lyapunov exponents, Ergod. Th. Dyn.Syst. 2 (1982), 109-124. 